\title{
Unsupervised Learning and Mapping of Brain fMRI Signals Based on Hidden Semi-Markov Event Sequence Models
}

\author{
Sylvain Faisan ${ }^{1,2}$, Laurent Thoraval ${ }^{1}$, Jean-Paul Armspach ${ }^{2}$, and \\ Fabrice Heitz ${ }^{1}$
}

1 Laboratoire des Sciences de l'Image, de l'Informatique et de la Télédétection, LSIIT/MIV - UMR CNRS 7005,

ENSPS, Bd. Sébastien Brant, F-67400 Illkirch, France

\{faisan, thoraval, heitz\}@ensps.u-strasbg.fr

http://lsiit.u-strasbg.fr

2 Institut de Physique Biologique,

Faculté de Médecine - UMR CNRS 7004,

4, Rue Kirschleger, F-67085 Strasbourg Cedex, France

armspach@ipb.u-strasbg.fr

http://ipb.u-strasbg.fr

\begin{abstract}
Most methods used in functional MRI (fMRI) brain mapping require restrictive prior knowledge about the shape of the active bloodoxygenation-level-dependent (BOLD) response, thus leading to suboptimal or invalid inference. To solve this problem, we propose to assess local neural activity in terms of time alignment between the sequence of BOLD dynamics changes of interest and an Hidden Semi-Markov Event Sequence Model (HSMESM) of brain activation. The topology of the HSMESM is built from the deterministic transitions of the input stimulation paradigm and its parameters are automatically and iteratively learned from all intracranial fMRI signals. The brain mapping results achieved by HSMESMs in language processing demonstrate the relevance of such models in BOLD fMRI, especially to cope with strong variabilities of the active BOLD signal across time, brain, experiments and subjects.
\end{abstract}

\section{Introduction}

Functional MRI extends conventional anatomical imaging to include localization and mapping of active brain areas engaged during perceptions, actions and cognitive tasks. Most existing methods used in fMRI brain mapping involve restrictive morphological assumptions about the shape of the BOLD signal observed at each voxel in response to an input stimulation block paradigm. These assumptions range from a simple on-off boxcar response, convolved or not with a predefined hemodynamic response function [2] [3] 4], to a more flexible fitting model formulated on a limited set of basis template functions as in the general linear model at the heart of SPM [5]. In addition, all these methods assume that 
the BOLD response is constant across stimulus (excluding habituation, learning and fatigue phenomenae) and, often, across brain areas, stimulation parameters, experimental sessions, and subjects. Consequently, fMRI data may be partially and misleadingly characterized leading to suboptimal or invalid inference.

To avoid prior morphological hypotheses, Thoraval et al. [1] recently proposed to resolve fMRI brain mapping based only on the relevant non-stationarities of the BOLD signal. In their approach, local neural activity is measured by scoring the relative alignment of the sequence of dynamics changes, detected in the corresponding BOLD signal, onto a deterministic model of the paradigm transition sequence. Despite the promising results obtained, the method suffers from the introduction of heuristic cost functions in the scoring process. In this paper, we propose to reformulate the scoring or evaluation problem within the statistical modeling framework of HSMESMs, a special class of Hidden SemiMarkov Models (HSMMs), to lead to a probabilistic brain activation model in place of the deterministic one mentionned above. The learning and generalizing abilities of HSMESMs prove to be of great interest in BOLD fMRI when dealing with the unknown shape and variability of the BOLD response.

\section{Hidden Semi-Markov Event Sequence Models}

This section presents only the salient features of HSMESMs within the general context of hidden Markov modeling. Because an HSMESM can be viewed as a special instance of an HSMM which in turn is an extension of the standard HMM to explicit modeling of state occupancy duration, the reader is referred to Rabiner's tutorial [6] for a rigorous presentation of the HMM formalism, and to the pioneering works of Ferguson [7], Russel et al. [8] and Levinson [9] for the understanding of durational modeling in HSMMs.

\subsection{Data Preprocessing, Event Sequence, Observation Sequence}

In an HSMESM approach, a preprocessing step detects and characterizes events of interest in the input process under analysis. Each time $t_{l}$ an event is detected, an observation, also called an event, $e_{l}$, is produced. Let $\tau_{O}=\{1,2, \ldots, T\}$ be the set of observation times and $O=o_{1} O_{2} \ldots o_{T}$ the corresponding observation sequence. Let $\tau_{E}=\left\{t_{1}=1, t_{2}, \ldots, t_{L}=T\right\}, \tau_{E} \subset \tau_{O}$, be the set of event detection times and $E=e_{1} e_{2} \ldots e_{L}$ the corresponding sequence of $L$ detected events. $e_{1}$ and $e_{L}$ are both fictive events introduced at the beginning and the end of $E$ for duration modeling purposes. Then, by definition, $O$ is built upon $E$ by inserting a null event, $\phi$, in $E$ each time $t \in \tau_{\bar{E}}=\tau_{O}-\tau_{E}$. The writing $o_{t}=\phi$ means a missing of observation occurred at time $t$. As an example, if $T=20$ and $\tau_{E}=\{1,3,6,12,15,20\}$, then $E=e_{1} e_{2} e_{3} e_{4} e_{5} e_{6}$ and $O=e_{1} \phi e_{2} \phi \phi e_{3} \phi \phi \phi \phi \phi e_{4} \phi \phi e_{5} \phi \phi \phi \phi e_{6}$. 


\subsection{Elements of an HSMESM}

Due to the detection based preprocessing, an HSMESM observation sequence is usually composed of true positive events $(t p e)$ mixed with false positive events (fpe) and missings of observation ( $n u l l$ ). Let $C-2$ be the number of tpe classes that characterize the process under study. Then, the state space of the associated HSMESM results from the union of two state subspaces, namely:

- $S_{t p e}=\left\{S_{i} ; i=1, \ldots, C\right\}: S_{i}$ is a standard Markov state producing tpe at times $t_{l} \in \tau_{E}$ according to its associated output observation probability density function $(p d f) b_{j}\left(o_{t_{l}}\right) . S_{1}$ et $S_{C}$ are the start and final states of the hidden process. They model the fictive events $e_{1}$ and $e_{L}$, respectively, with the arbitrary settings $b_{1}\left(o_{t_{1}}=e_{1}\right)=1$ and $b_{C}\left(o_{t_{L}}=e_{L}\right)=1$.

$-S_{f p e / \text { null }}=\left\{S_{i j} ; i, j=1, \ldots, C\right\}: S_{i j}$ is a semi-markovian state interleaved between $S_{i}$ and $S_{j} . S_{i j}$ is occupied $d-1$ times when the model transits from $S_{i}$ to $S_{j}$, with an inter-tpe state duration $d, d \geq 1$, between $S_{i}$ et $S_{j}$ explicitly modeled by the duration $p d f p_{i j}(d)$ in accordance with the semi-markovian nature of $S_{i j}$. $S_{i j}$ produces fpe or null at times $t \in \tau_{\bar{E}}$ according to its composite output observation $p d f b_{i j}^{+}\left(o_{t}\right)=b_{i j}\left(e_{l}\right) \cdot e_{i j 1} \cdot \mathbb{1}_{\tau_{E}}(t)+e_{i j 2} \cdot \mathbb{1}_{\tau_{\bar{E}}}(t)$ $\left(\mathbb{1}_{X}(t)=1\right.$ if $t \in X, 0$ otherwise) built on the latent Bernoulli process of parameter $e_{i j 1}=1-e_{i j 2}$.

To be completely specified, an HSMESM needs to define the $C$ parameter, the transition probability matrix $A=\left\{a_{i j}\right\}$, the observation $p d f_{\mathrm{s}} B=\left\{b_{j}().\right\}$ and $B^{\prime}=\left\{b_{i j}().\right\}$, the durational $p d f \mathrm{~s} D=\left\{p_{i j}().\right\}$ and the emission matrix $E=$ $\left\{e_{i j 1}\right\}$. Hereafter, we use the compact notation $\lambda=\left(A, B, B^{\prime}, D, E\right)$ to denote an HSMESM.

From a generative point a view, an HSMESM behaves as follows. In $S_{1}$ at $t_{1}$, the model emits $e_{1}$. At some $t_{k}, 1 \leq k<L$, in $S_{i}$, it selects the next state $S_{j}$ to visit according to the transition probability distribution $\left\{a_{i j}\right\} . S_{j}$ is actually visited at $t_{l}=t_{k}+d, l>k$, with $d$ randomly drawn according to the inter-tpe distribution $p_{i j}(d)$. Before reaching $S_{j}$, the model remains $d-1$ times in $S_{i j}$ to emit fpe or null according to $b_{i j}^{+}($.$) . This procedure is repeated until reaching$ $S_{C}$ at $t_{L}=T$ to emit $e_{L}$.

The topological representation of an HSMESM is depicted in Fig. 11 b. Shaded circles represent tpe states while bicolor boxes in between correspond to $\mathrm{fpe} / \mathrm{null}$ states. The equivalent standard markovian representation of an HSMESM can be obtained by expanding the semi-Markov state $S_{i j}$ into two parallel state delay lines, as shown in Fig. 1k, one composed of $D-1$ standard HMM states $S_{i j}^{o, d}$ with an associated output $p d f b_{i j}($.$) , the other composed of D-1$ standard HMM states $S_{i j}^{\varnothing, d}$ without any output $p d f$. $D$ denotes the maximum duration allowed between two successive tpe. When transiting from $S_{i}$ to $S_{j}$, the hidden process swaps from one delay line to the other. For instance, observing the subsequence of Fig. 1a is equivalent at the hidden process level to occupy $S_{i}, S_{i j}^{\phi, 7}, S_{i j}^{o, 6}$ and so on until reaching $S_{j}$. An HSMESM can be seen as a special class of HSMM since setting $e_{i j 1}=1$ and $b_{i j}(.) \equiv b_{j}($.$) in Fig. 11c leads back to Levinson's equivalent$ standard markovian representation of a semi-Markov state. 
a)

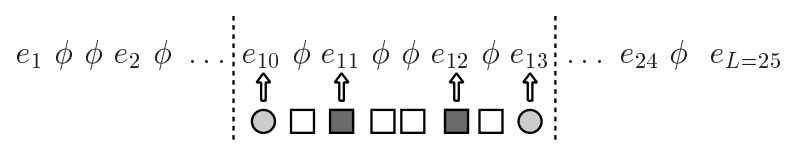

b)

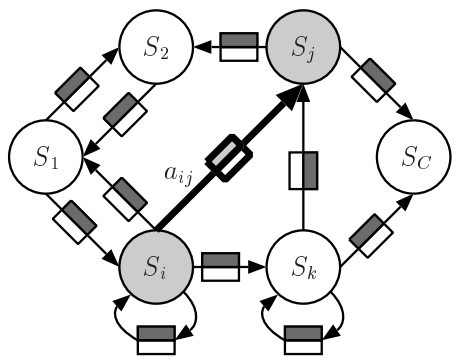

c)

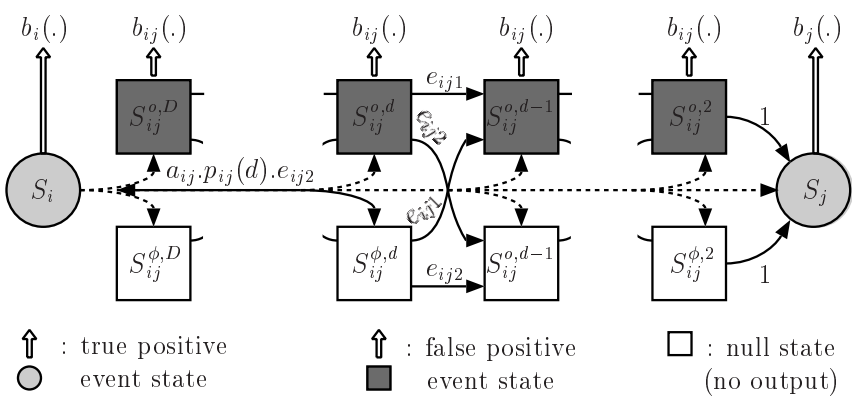

Fig. 1. HSMESM example: a) output observation sequence with some of the corresponding visited states, b) topological representation, c) equivalent standard markovian representation of the state triplet $\left\{S_{i}, S_{i j}, S_{j}\right\}$ (see text).

\subsection{Algorithmic Aspects}

The three basic problems of HMMs or HSMMs, namely evaluation, learning and decoding [6], arise equally within an HSMESM modeling framework. They can be solved similarly based on adapted versions of the Forward-Backward, BaumWelch and Viterbi algorithms, respectively. They are not presented in this paper. However, it can be stressed that the Forward, Backward and Viterbi variables, when implemented in an HSMESM analysis framework, are calculated only for the detection time series $\left\{t_{l}\right\}$, thus reducing substantially the computation load and the memory requirements as soon as the number of detected events, $L$, becomes much less than the number of observations, $T$.

\section{Unsupervised Learning and Mapping of fMRI Signals}

\section{1 fMRI Data Preprocessing}

The events to be detected here are the significant dynamics changes of the local BOLD response. To this aim, the wavelet-based preprocessing approach of Tho- 
raval et al. [1] is used. Unlike their approach, only the "off-on" dynamics changes of interest are tracked. Indeed, due to a locally smoothed shape of the BOLD response or due to an unsustained cerebral response, or both, the temporal localization of the "on-off" dynamics changes of interest is made too unprecise, leading to excessive noise in terms of time alignment onto the paradigm transitions.

\subsection{Brain Activation HSMESM}

At the hidden process level, $C-2$ tpe states $S_{i}$ are used, one for each "off-on" paradigm transition, with a state index $i$ reflecting the order of appearance of the transition in the paradigm. The start and final tpe states $S_{1}$ and $S_{C}$ are then added and a semi-Markov state $S_{i j}$ inserted between each valid tpe state pair $\left(S_{i}, S_{j}\right)$. A left-right topology is selected for the chain with the additional contraints $a_{i i}=0, \forall i$, and $a_{i j}=0$ if $j>i+\Delta$, with $\Delta \sim \frac{C}{2}$, to avoid an active signal jumps too many successive transitions at once. A typical chain topology of a brain activation HSMESM is depicted in Fig. 2 with $\Delta=2$ and $C=5$. For simplicity of the model and consistency in the reestimation of its parameters, all observation and durational $p d f$ s are specified as one-dimensional gaussians. Finally, to reduce the amount of parameters to be estimated, the concept of parameter tying [6] is extensively used.

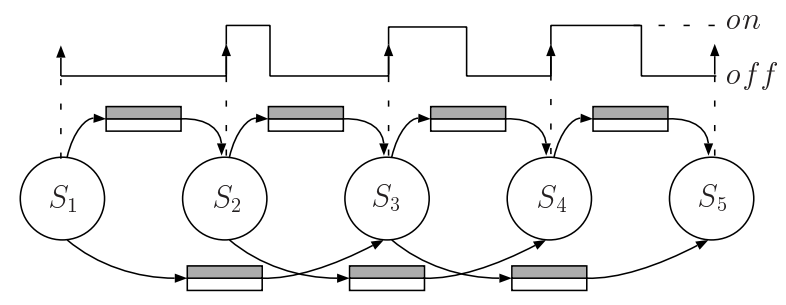

Fig. 2. State chain example of a brain activation HSMESM : a) paradigm off-on transition sequence with the fictive beginning and end off-on transitions, b) associated hidden state chain with the tpe (circles) and fpe/null (boxes) states.

\subsection{Unsupervised Learning and Mapping}

Unsupervised learning and mapping of brain active signals are performed jointly in the sake of a brain activation HSMESM well-suited for the fMRI sequence under analysis. The procedure consists in building iteratively a series of refined brain activation models $\left\{\lambda_{i}\right\}$ along with a series of active signal sets $\left\{S_{i}\right\}$. Composed of all the signals declared active by $\lambda_{i}, S_{i}$ is used as learning set for $\lambda_{i+1}$. Both series converge respectively to $\lambda_{\text {opt }}$ and $S_{\text {opt }}$ in that learning from 


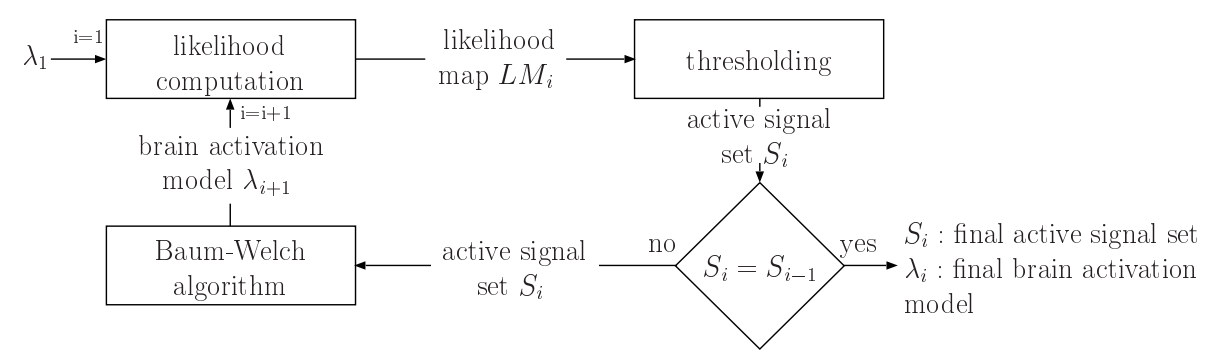

Fig. 3. Learning-Mapping procedure of a brain activation HSMESM

$S_{o p t}$ yields $\lambda_{\text {opt }}$ which in turn declare active $S_{o p t}$. The overall procedure is depicted in Fig. 3. The initial model $\lambda_{1}$ is derived from simple statistics performed on the active signal set $S_{0}$ of all intracranial event sequences. The likelihood map $L M_{i}$ is made of the voxel likelihoods $l=P\left(O \mid \lambda_{i}\right)$ for which a $p$-value $p=\int_{l}^{+\infty} P\left(x \mid H_{0}\right) d x$ is calculated, where $P\left(x \mid H_{0}\right)$ is the $p d f$ of the likelihood $x$ under the null hypothesis $H_{0}$ of cerebral inactivity. This distribution is inferred experimentally from a large set of random observation sequences, representative of chance, obtained by a random mixing procedure applied to the intracranial event sequences. The $p$-value map associated with $L M_{i}$ is thresholded to yield the active signal set $S_{i}$. If $S_{i}$ coincide with $S_{i-1}$, the learning-mapping procedure is stopped with $S_{i}$ as the final brain activation map. Otherwise, $S_{i}$ is reused to estimate $\lambda_{i+1}$ based on the Baum-Welch algorithm. It must be noted that the above procedure converged for all fMRI sequences tested, and independtly of the initial model $\lambda_{1}$, provided $\lambda_{1}$ is a weakly-constrained brain activation HSMESM.

\section{Results and Discussion}

fMRI studies have been conducted to map the cortical areas involved in language processing on 16 healthy volunteers using a two-condition task [10]. Images were acquired with a $2 \mathrm{~T}$ whole body S200 Bruker MRI system with a head volume coil. Each fMRI sequence is composed of 145 3D images obtained with echoplanar imaging (EPI) using an axial slice orientation (32 slices, 64x64 pixels, voxel size $=4 \times 4 \times 4 \mathrm{~mm}, \mathrm{TE}=10 \mathrm{~ms}, \mathrm{TR}=5 \mathrm{~s})$. All images are registered to the first image in the series. A spatial prefiltering is then performed to increase the signal to noise ratio. Activation maps obtained with the HSMESM approach are compared to the ones obtained by the $t$-test (Fig. 4a-d). The thresholded HSMESM map of Fig. 4r indicates four brain areas implicated in the language task. These are also detected by the $t$-test, as shown in Fig. 4d, except for Region 3, thereby illustrating the ability of the HSMESM method to exhibit additional true activated areas in the brain. Moreover, signals declared active outside the language areas by the HSMESM method, as Region 5 in Fig. 4 $\mathrm{c}$, do not correspond to false alarms but to induced secondary activities. Generally speaking, true active fMRI signals undetected by the $t$-test present either impulsive noise, baseline drift or 

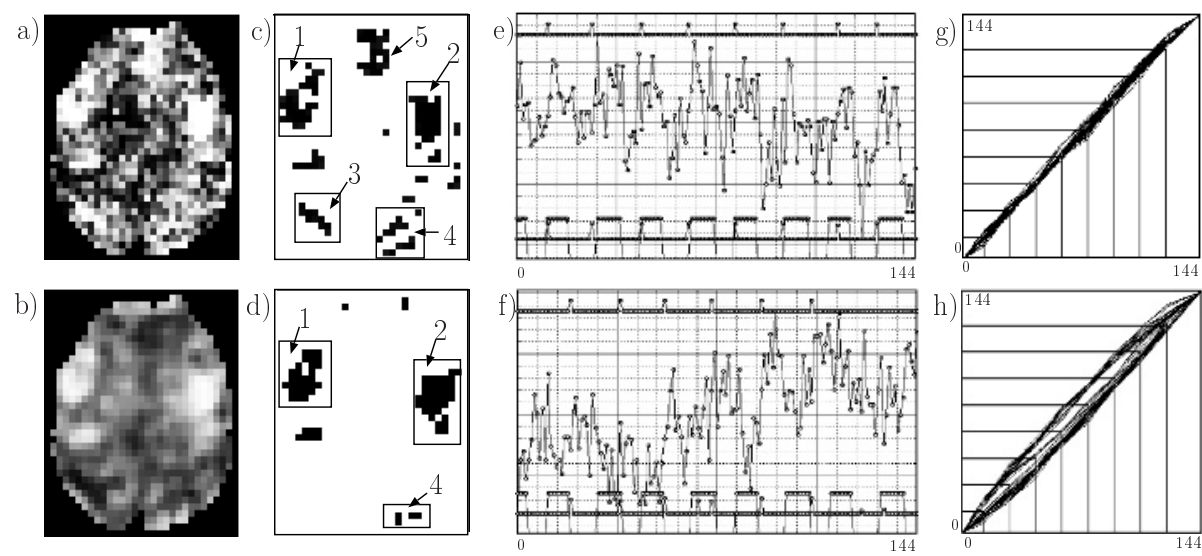

Fig. 4. Results: a,b) activition maps in terms of $p$-value $(p=0-1$ (white-black)) obtained with the HSMESM method (a) and the $t$-test method (b), c,d) corresponding thresholded maps $(p=0.05)$, e) active signal from Region 1, detected by both methods, f) active signal from Region 3, detected by the HSMESM method only, with, for e) and f), from bottom to top: stimulation paradigm with off-on transitions, dynamics changes detected in the raw fMRI signal, raw fMRI signal, tpe detection time series after Viterbi decoding, g,h) matching plots of the tpe detection time series vs. the off-on transition time series for a fMRI signal of Region 1 (g) and Region 5 (h)

activation delays along with low SNRs, as shown in Fig. 屃. They may also correspond to emerging or vanishing activities, that is, to unsustained activities over the fMRI experiment. Finally, both matching plots of Fig. 4s-h illustrate another promising aspect of the HSMESM approach in BOLD fMRI. They are obtained for two different regions of interest, namely Region 1 and Region 5, by plotting for each voxel in the corresponding region, the tpe detection time series $\left\{\hat{t}_{i}\right\}, \hat{t}_{i}$ in $\tau_{E}$, decoded by the Viterbi algorithm onto the brain activation HSMESM, against the off-on transition time series $\left\{t_{i}\right\}$ of the stimulation paradigm. The subplot of Fig. 45 is typical of an in-phase cerebral response for Region 1 with respect to the stimulation paradigm. Instead, the half-opened shape depicted in Fig. 4h is representative of an anti-phase cerebral response for Region 5, with an underlying brain activation model not willing to decide whether the active fMRI signal is in advance or late with respect to the paradigm transition sequence. Finally, note that regarding computational issues, the processing time needed to obtain an HSMESM activation map from a given input fMRI sequence, spatial registration excluded, was about 10 minutes on a PC Pentium 4, 1.7 GHz.

\section{Conclusion and Future Work}

A new statistical method for learning and mapping active signals in functional MRI of the human brain has been presented. Unlike other methods, no prior restrictive morphological assumptions are required. Neural activity is assessed only 
on the BOLD dynamics changes of interest detected at each voxel. The event sequence they form is scored using a statistical brain activation hidden semiMarkov event sequence model built from the deterministic sequence of stimulation paradigm transitions, and statistically trained from all intracranial fMRI signals.

The brain mapping results achieved by the HSMESM method are encouraging. They first demonstrate the relevance of such statistical models in BOLD fMRI to take into account the variabilities of the BOLD active signal across time, space, experiments and subjects. They also validate the time locking principle between stimulation and response introduced by Thoraval et al. [1 in human brain mapping. Finally, they lead to the design of new fMRI functionalities from HSMESM Viterbi decoding such as selecting, focusing and analyzing specific activation modes.

The proposed method has now to be compared further. In addition, we plan to introduce neighbouring spatial information in the measure of the local neural activity by extending the HSMESM framework to the case of hidden semiMarkov multiple event sequence models.

\section{References}

1. Thoraval, L., Armspach J.-P., Nammer I.: Analysis of brain functional MRI time series based on continuous wavelet transform and stimulation-response coupling distance. In Proc. MICCAI Conf., (2001) 881-888.

2. Friston, K.J., Jezzard, P., Turner, R.: The analysis of functional MRI time-series. Human Brain Mapping, 1 (1994) 153-171.

3. Lange, N., Zeger, S.L.: Non-linear fourier time series analysis for human brain mapping by functional magnetic resonance imaging. Applied Statistics, Journal of the Royal Statistical Society, Series C, 46 (1997) 1-29.

4. Rajapakse, J.C., Kruggel, F., Maisog, J.M., von Cramon, D.Y.: Modeling Hemodynamic Response for Analysis of Functional MRI Time-Series. Human Brain Mapping, 6 (1998) 283-300.

5. Frackowiak, R.S.J., Friston, K.J., Frith, C.D., Dolan, R.J., Mazziota, J.C (editors): Human Brain Function. Academic Press USA, 1997.

6. Rabiner, L.R.: A Tutorial on Hidden Markov Models and Selected Applications in Speech Recognition. Proceedings of the IEEE, 77 (1989) 257-286.

7. Ferguson, J.D.: Variable duration models for speech. In Proc. Symposium on the Application of Hidden Markov Models to Text and Speech, (1980) 143-179.

8. Russell, M., Moore, R.: Explicit modelling of state occupancy in Hidden Markov Models for automatic speech recognition. In Proc. ICASSP, (1985) 5-8.

9. Levinson, S.E.: Continuously variable duration hidden Markov models for automatic speech recognition. Computer Speech and Language, 1 (1986) 29-45.

10. Metz-Lutz, M.-N., Namer, I.J., Gounot, D., Kleitz, C., Armspach, J.-P., Kehrli, P.: Language functional neuro-imaging changes following focal left thalamic infarction. Neuroreport, 11 (2000) 2907-2911. 